\title{
XVIII. Ueber Grassmann's Methode der Axen- darstellung und ihre Anwendung auf die Lösung gewisser krystallographischer Probleme.
}

\author{
Von \\ W. J. Lowis in Cambridge, England.
}

(Mit 2 Textfiguren).

Grassmann bezog in sciner 1829 publicirten $\Lambda$ bhandlung * Zur physischen Krystallographie und geometrischen Combinationslehre einen Krystall auf drei Flächennormalen - Strahlen — als Bezugsaxen, wobei die Parameter die den $\Lambda$ xen parallelen Kanten eines Parallelepipeds sind, dessen Diagonale eine bestimmte Länge einer bekannten Normale ist, welche mit keinem Paare der Axenstrahlen coplanar ist. Irgend eine andere Normale wird alsdann dargestellt durch die Diagonale eines ähnlich gebildeten l'arallelepipeds, welches zu Kanten parallel den Axen Längen besitzt, welche rationale Vielfache der Parameter sind. Grassmann gab keine Formeln an, durch welche allgemein die Indices von Flächennormalen aus Messungen der Winkel am Krystalle gefunden werden, noch solche zur Bestimmung der Winkel aus den Krystallelementen und Indices. Frankenheim (Crelle's Journal für Mathematik 1832, 8, 178) gab einen Ausdruck für den Cosinus des Winkels zwischen zwei beliebigen Strahlen, aber dieser Ausdruck hat nicht die jetzt gebräuchliche Form. Miller in seiner Abhandlung über die Methode (Proc. Camb. Phil. Soc. 1868, 2, 75), beschränkt sich auf einigc allgemeine, auf Zonen bezügliche Probleme. Der Platz, welcher mir in mcinem * Treatise on Crystallography 1899 « für den Gegenstand zur Verfügung war, erlaubte mir nur, die Beziehung anzugeben, welche die Strahlenparameter mit denen eines auf Zonenaxen bezogenen Krystalles verbindet. Aber die Neigung eines beliebigen Strahles zu den Bezugsaxen und zu irgend einem anderen Strahl kann leicht gefunden werden auf dem Wege, welcher für dic ähnlichen Relationen zwischen der Richtung einer Linie und solchen Axen, wolche Kanten des Krystalles sind, in Kap. XIX, $\Lambda$ rts. 
$9,10,11,13$ und 14 meines Treatise benutzt worden ist. Dic Beziehungen, welche sich ergeben, wenn der Krystall auf Axenstrahlen bezogen wird, sind unmittelbar anwendbar auf die durch Goniometermessung erhaltenen Winkel und sind in vieler Hinsicht einfacher, als die in den oben crwïhnten $\Lambda$ rtikeln gegebenen Relationen. J)a ausserdem (irassmann's Axendarstellung manche elegante Ausdrücke liefert, welche auf die Auflösung von einigen bei der Bestimmung der Flächensymbole und Winkel der Krystalle rorkommenden Problemen anwendbar sind, so habe ich gredacht, es möchte sich nülzlich erweisen, einige der Ilauptrelationen aufzustellen.

Wir werden in Folgendem stets annehmen, dass dieselben drei Flächen zur Bestimmung des Axensystems ausgewählt werden, indem die Axenstrahlen deren Normalen und die gewöhnlichen Axen deren Schnittkanten darstellen. Wir werden mit $O x, O y$ und $O \approx$ die Strahlen bezeichnen, welche respective senkrecht zu den Flächen $(100),(010)$ und $(001)$ sind. $O G$ sei der Parameterstrahl ( 111 ), und wir werden ihn senkrecht zu der Fläche (111), bezogen auf Zonenaxen, annehmen. Die Strahlenparameter werden mit $\boldsymbol{a}, \boldsymbol{b}, \boldsymbol{c}$ und die Winkel zwischen den $\Lambda$ xenstrahlen mit $\alpha, \beta, \gamma$ bezeichnet. Die entsprechenden Parameter und Winkel, wenn der Krystall auf Zonenaxen bezogen wird, scien $a, b, c, a_{1}, \beta_{1}, \gamma_{1}$. Ein beliebiger Strahl $(h k l)$ ist alsdann gegeben durch die Gleichungen

$$
\stackrel{x}{l \ddot{\boldsymbol{c}}}=\frac{y}{k \dot{\boldsymbol{b}}}=\frac{\approx}{l \boldsymbol{c}}
$$

und die Strahlenparameter sind mit den auf Zonenaxen bezüglichen verbunden durch die Gleichungen

$$
\frac{\boldsymbol{a} a}{\sin \alpha}=\frac{\boldsymbol{b} b}{\sin \beta}=\frac{\boldsymbol{c} c}{\sin \gamma}
$$

oder die mit ihnen äquivalenten

$$
\underset{\sin \alpha_{1}}{\boldsymbol{a} a}=-\frac{\boldsymbol{b} b}{\sin \beta_{1}}=\frac{c c}{\sin \gamma_{1}} .
$$

Salz I. Es ist die Lünge eines gegebenen strables (llkl) durch die Indices, die Strahlenparameler und dic Winkel zwischen den $\mathbf{A x e n s t r a h l e n ~ a u s z u d r u ̈ c k e n . ~}$

Es sei $O L P N$ das Parallelepiped, welches $O P$ als Diagonale und als Kanten parallel den Axen die Längen hat:

$$
\begin{gathered}
O L=F M=h \boldsymbol{a}, O M=L F=k \boldsymbol{b}, \\
O N=P F^{\prime}=l \boldsymbol{c} .
\end{gathered}
$$

Die Neigung von $O I^{\prime}$ zu den $\Lambda$ xen $O x$,

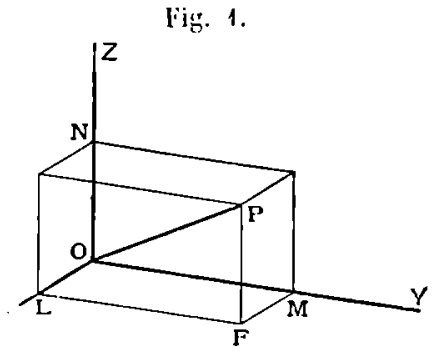
$O y, O x$ soll mit $\lambda$, resp. $\mu, \nu$ bezeichnct werden. 
Da die summe der orthogonalen I'rojectionen der drei Kanten $O L$, $L F^{\prime}$ und $F^{\prime} P^{\prime}$ auf $O P^{\prime}$ gleich der Länge $O P$ sein muss, so haben wir

$$
\begin{array}{r}
O P=O L \cos \lambda+L F \cos \mu+F P \cos \nu \\
=h \boldsymbol{a} \cos \lambda+k \boldsymbol{b} \cos \mu+l \boldsymbol{c} \cos \nu .
\end{array}
$$

Ferner erhalten wir, wenn wir die orthogonalen Projectionen von $O P^{P}$ und der drei crwähnten Kanten des Parallelepipeds auf jeden der $\Lambda$ xenstrahlen abwechsclnd nehmen, dic folgenden Gleichungen:

$$
\left.\begin{array}{rl}
O P \cos \lambda=O L+L F \cos \gamma+F P \cos \beta & =h \boldsymbol{a}+l \boldsymbol{b} \cos \gamma+l \boldsymbol{c} \cos \beta \\
O P \cos \mu & =h \boldsymbol{a} \cos \gamma+l \boldsymbol{b}+l \boldsymbol{c} \cos \alpha \\
O P \cos \nu & =h \boldsymbol{a} \cos \beta+l \boldsymbol{b} \cos \boldsymbol{\alpha}+l \boldsymbol{c}
\end{array}\right\} .(\boldsymbol{b})
$$

Multiplicirt man beide Seiten von (4.) mit $O P$ und führt die Werthe von $O P \cos \lambda$, ctc. aus (j) ein, so crhült man:

$$
\begin{aligned}
O P^{2} & =h \boldsymbol{a}(h \boldsymbol{a}+k \boldsymbol{b} \cos \gamma+l \boldsymbol{c} \cos \beta)+k \boldsymbol{b}(h \boldsymbol{u} \cos \gamma+l \boldsymbol{b}+l \boldsymbol{c} \cos \alpha) \\
& +l \boldsymbol{c}(h \boldsymbol{c} \boldsymbol{c o s} \beta+k \boldsymbol{b} \cos \alpha+l \boldsymbol{c})=\Sigma h^{2} \boldsymbol{a}^{2}+2 \Sigma l \boldsymbol{b} \boldsymbol{c} \cos \alpha .
\end{aligned}
$$

Satz II. Es sind die Richtungscosinus eines beliebigen Strahles und die Relation gesucht, welche zwischen ihnen und den Winkeln zwischen den Axenstrahlen besteht.

Die Richtungscosinus werden gefunden, indem man in (5) den in $(6)$ gegebenen Werth von $O P^{\prime}$ einsetzt, dieselben sind daher:

$$
\begin{aligned}
& \cos \lambda=\frac{h \boldsymbol{a}+k \boldsymbol{b} \cos \gamma+l \boldsymbol{c} \cos \beta}{\sqrt{\boldsymbol{\Sigma} h^{2}} \overline{\boldsymbol{c}}^{2}+2 \boldsymbol{\Sigma} k l \boldsymbol{b} \boldsymbol{c} \cos \alpha}
\end{aligned}
$$

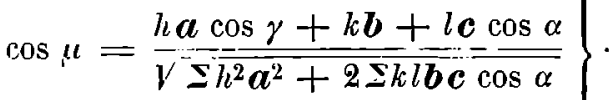

$$
\begin{aligned}
& \cos \nu=\frac{h \boldsymbol{a} \cos \beta+k b \cos a+l \boldsymbol{c}}{\sqrt{\Sigma h^{2} a^{2}+2 \boldsymbol{\Sigma} k l b c \cos \alpha}}
\end{aligned}
$$

Dic Gleichungen (4) und (5) gelten für einen beliebigen Strahl. Eliminirl man daher $O I, h \boldsymbol{a}, k \boldsymbol{b}, l \boldsymbol{c}$ aus den vier Gleichungen, so erhält man die zwischen den lichtungscosinus bestehende lelation; diese ist

$$
A_{1}=\left|\begin{array}{cccc}
1 & \cos \lambda & \cos \mu & \cos \nu \\
\cos \lambda & 1 & \cos \gamma & \cos \beta \\
\cos \mu & \cos \gamma & 1 & \cos \alpha \\
\cos \nu & \cos \beta & \cos \alpha & 1
\end{array}\right|=0 .
$$

Satz III. Es sind die Indices eines beliebigen Strahles durch die Winkel, welche derselbe mit den $\Lambda$ xenstrahlen bildet, auszudrücken.

Löst man die Gleichungen (ö) für $h \boldsymbol{a}, k \boldsymbol{b}, l \boldsymbol{c}$ auf, so erhält man

$$
h a \div\left|\begin{array}{ccc}
\cos \lambda & \cos \gamma & \cos \beta \\
\cos \mu & 1 & \cos \alpha \\
\cos \nu & \cos \alpha & 1
\end{array}\right|=k b \div\left|\begin{array}{ccc}
1 & \cos \lambda & \cos \beta \\
\cos \gamma & \cos \mu & \cos \alpha \\
\cos \beta & \cos \nu & 1
\end{array}\right|
$$


$=l c \div\left|\begin{array}{ccc}1 & \cos \beta & \cos \lambda \\ \cos \gamma & 1 & \cos u \\ \cos \beta & \cos \alpha & \cos \nu\end{array}\right|=O O^{\prime} \div\left|\begin{array}{ccc}1 & \cos \gamma & \cos \beta \\ \cos \gamma & 1 & \cos \alpha \\ \cos \beta & \cos \alpha & 1\end{array}\right|$.

Ferner erhält man, wenn man den Werth von $O l^{\prime}$, wie or in (4) gegeben ist, in die Gleichungen (:3) cinsetzt, dic folgenden, unter Umsländen verwendbaren Glcichungen:

$h \boldsymbol{a} \sin ^{2} \lambda+k \boldsymbol{b}(\cos \gamma-\cos \lambda \cos \boldsymbol{\mu})+l \boldsymbol{c}(\cos \beta-\cos \lambda \cos \mu)=0$

$h \boldsymbol{a}(\cos \gamma-\cos \lambda \cos \boldsymbol{u})+k \boldsymbol{b} \sin ^{2} \boldsymbol{u}+\boldsymbol{l} \boldsymbol{c}(\cos \alpha-\cos \boldsymbol{\mu} \cos \nu)=0$ $\left.h \boldsymbol{a}(\cos \beta-\cos \lambda \cos \nu)+k \boldsymbol{b}(\cos \alpha-\cos u \cos \nu)+l \boldsymbol{c} \sin ^{2} \nu=0\right)$

Satz IV. Gesucht die Gleichung der zu einem Strahle $(h k l)$ senkrechten Ebene, d. h. der liläche $(l k l)$.

Die Ebene möge durch den Punkl $P$ gehen, d. h. den Endpunkt der Diagonalen des Parallelepipeds mit den Kanten $h \boldsymbol{a}, k \boldsymbol{b}, l \boldsymbol{c}$, und der Strahl mügc mit den $\Lambda$ xenstrahlen die Winkel $\lambda, ", \nu$ bilden.

Die Summe der orthogonalen Projectionen der Coordinaten $x, y, z$ eines beliebigen in der Jjbene liegenden Punktes auf $O P$ ist glcich $O P$.

$$
\therefore O P=x \cos \lambda+y \cos \mu+\approx \cos \nu \text {. }
$$

Führt man dic Werthe von $\cos \lambda, \cos \mu, \cos \nu$ aus (:3) und den Werllı von $O P^{2}$ aus (6) ein, so crhält man

$$
\begin{array}{r}
(x-h \boldsymbol{c})(h \boldsymbol{a}+k \boldsymbol{b} \cos \gamma+l \boldsymbol{c} \cos \beta)+(y-k \boldsymbol{b})(h \boldsymbol{a} \cos \gamma+l i \boldsymbol{b}+l \boldsymbol{c} \cos \alpha) \\
+(\boldsymbol{c}-l \boldsymbol{c})(h \boldsymbol{a} \cos \beta+k \boldsymbol{b} \cos \alpha+l \boldsymbol{c})=0
\end{array}
$$

Dic parallele Ebcne durch den Ursprung ist:

$$
\begin{gathered}
x(h \boldsymbol{a}+k \boldsymbol{b} \cos \gamma+l \boldsymbol{c} \cos \beta)+y(h \boldsymbol{a} \cos \gamma+k \boldsymbol{b}+l \boldsymbol{c} \cos \alpha) \\
+x(h \boldsymbol{a} \cos \beta+k \boldsymbol{b} \cos \alpha+l \boldsymbol{c})=0 .
\end{gathered}
$$

Satz V. Gesucht der Winkel $\varphi$ zwischen zwei beliebigen Strahlen $(h l l)$ und $\left(h_{1} k_{1} l_{1}\right)$.

$O P$ in Fig. 1 sei der Strahl $(h k l)$, und seine Neigung zu den $A$ xenstrahlen sei $\lambda$ resp. $\mu, \nu$. Dann gelten für ihn die in den vorhergehenden Sätzen gegebenen Relationen. Wenn man annimml, dass der Strahl $\left(h_{1} l_{1} l_{1}\right)$ durch die Diagonale $O P_{1}$ cines ähnlichen Parallelepipeds gegeben sei und dass scine Richtungswinkel $\hat{\lambda}_{1}, \mu_{1}, \nu_{1}$ scien, so muss die orthogonale Projection von $O P$ auf $O P_{1}$ gleich der Summe aus den Projectionen der Kanten $O L, L F, F^{\prime} P$ auf $O I_{1}^{\prime}$ sein.

Daher: $\quad O P \cos \varphi=O L \cos \lambda_{1}+L F \cos \mu_{1}+F P \cos \nu_{1}$

$$
=h \boldsymbol{a} \cos \lambda_{1}+k \boldsymbol{b} \cos \mu_{1}+l \boldsymbol{c} \cos \nu_{1} \text {. }
$$

Aber $\cos \lambda_{1}, \cos \mu_{1}, \cos \nu_{1}, O P_{1}$ sind miteinander verbunden durch Gleichungen, welche ähnlich den in (5) gegelsenen sind. Multiplicirt man beide Seiten von (13) mit $O I_{1}^{\prime}$ und erselzl $O I_{1}^{\prime} \cos \lambda_{1}$ ele. durch ihre Werthe, so crbïlt man: 


$$
\begin{aligned}
& O P . O I_{1}^{\prime} \cos \varphi=h \boldsymbol{a}\left(h_{1} a+l_{1} b \cos \gamma+l_{1} c \cos \beta\right) \\
& +l_{i} b\left(h_{1} a \cos \gamma+l_{1} b+l_{1} c \cos \alpha\right)+l c\left(l_{1} a \cos \beta+k_{1} b \cos \alpha+l_{1} c\right) .
\end{aligned}
$$

Folglich:

$$
\cos \varphi=\frac{\Sigma h h_{1} a^{2}+\Sigma b c\left(k l_{1}+l l_{1}\right) \cos \alpha}{v \Sigma h^{2} a^{2}+2 \Sigma k l b c \cos \bar{\alpha} \quad v \Sigma h_{1}^{2} a^{2}+2 \Sigma h_{1} l_{1} b c \overline{c o s} \alpha} .
$$

Dieser Ausdruck ist einfacher und übersichtlicher als irgend einer der Ausdrïcke (44) und (45) auf S. 569 und 870 meines Treatise on Crystallography. Ferner kann er leicht in jeden von ihnen umgewandelt werden mil llïlfe der Relationen (2). So ist, um (47) zu erhalten, für $\boldsymbol{a}, \boldsymbol{b}, \boldsymbol{c}$ nur nöthig ihre Werthe aus (2) einzusetzen:

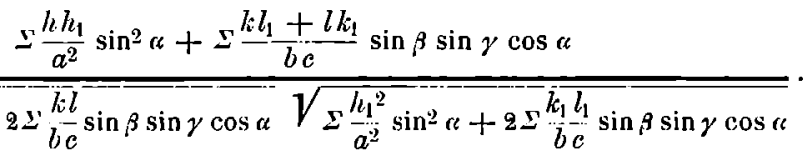

Glcichung (4.4) wird durch eine ähnliche Substitution gefunden mit llülfe der Relationen eines sphärischen Dreiecks:

$$
\operatorname{Cos} \alpha=\cos \beta \cos \gamma+\sin \beta \sin \gamma \cos \left(A=\pi-\alpha_{1}\right) \text {, etc. }
$$

Wir werden nun sehen, wie diese Methode auf die Lösung krystallographischer Probleme angewendet werden kann. Es möge ein trikliner Krystall angenommen und verlangt werden, die linearen Elemente aus cinigen bckannten Winkeln zu finden. Es sollen die Buchstaben und die Bezeichnung von S. 161 meines Treatise angenommen werden, und es sei die Zone $[B L C]=[010,001]$ als bekannt vorausgesetzt. Durch Einführung der Indices $0,1,1$ von $L$ in die 1. und 2. Gleichung (7) crliält man:

und

$$
\begin{gathered}
\cos (A L=100: 011)=\frac{\boldsymbol{b} \cos \gamma+\boldsymbol{c} \cos \beta}{\sqrt{\boldsymbol{b}^{2}+\boldsymbol{c}^{2}+2 \boldsymbol{b} \boldsymbol{c} \cos \alpha}} \\
\sec ^{2}(D=010: 011)=\frac{\boldsymbol{b}^{2}+\boldsymbol{c}^{2}+2 \boldsymbol{b c} \cos \alpha}{(\boldsymbol{b}+\boldsymbol{c} \cos \alpha)^{2}} \\
\therefore \operatorname{tg}^{2} D=\sec ^{2} D-1=\frac{\boldsymbol{c}^{2}\left(1-\cos ^{2} \alpha\right)}{(\boldsymbol{b}+\boldsymbol{c} \cos \alpha)^{2}} \\
\therefore \operatorname{tg} D=\frac{\boldsymbol{c} \sin \alpha}{\boldsymbol{b}+\boldsymbol{c} \cos \alpha}
\end{gathered}
$$

ferner

$$
\begin{aligned}
\frac{\sin D}{c \sin \alpha} & =\frac{\cos D}{\boldsymbol{b}+\boldsymbol{c} \cos \alpha}=\frac{\sin (\alpha-D)}{\boldsymbol{b} \sin \alpha} \\
\therefore \frac{\boldsymbol{b}}{\boldsymbol{c}} & =\frac{\sin (\alpha-D)}{\sin D}=\frac{\sin D}{\sin D}
\end{aligned}
$$

J)iese Gleichung, welche das Parameterverhailtniss $\frac{\boldsymbol{b}}{\boldsymbol{c}}$ liefert, giebt 
Grassmann S. 138; aber da er versucht, das Verhältniss mil den $\Lambda \mathrm{b}$ stïnden zwischen aufeinander folgenden Partikeln zu verbinden, so differilt sein Ausdruck ein wenig von dem obigen. Li kann mittelst der Relationen (2) leicht in die Gleichungen umgewandelt werden, welche die Parameter auf den correspondirenden Zonenaxen liefern. Der letztere Ausdruck ist:

$$
\frac{c \sin }{b \sin } \frac{\beta}{\gamma}=\frac{\sin D}{\sin D}
$$

oder

$$
\frac{b}{c}=\frac{\sin B L}{\sin C L} \cdot \frac{\sin C A}{\sin A B} .
$$

Durch Einführung der Werthe (101) und (110) in (7) erhalten wir correspondirende Ausdrückc für die anderen Pitramelerverhältnisse. Iiese sind:

$$
\frac{c}{a}=\frac{\sin E}{\sin E}, \text { und } \frac{a}{b}=\frac{\sin F r}{\sin F} .
$$

Ferner, wenn $L$ gleich $0 \bar{k} l$, so erhalten wir aus der zwciten Gileichung von (7)

$$
\cos B L,=\frac{l \boldsymbol{b}+l \boldsymbol{c} \cos \alpha}{\sqrt{k^{2} \boldsymbol{b}^{2}}+l^{2} \boldsymbol{c}^{2}+2 k l \boldsymbol{b} \boldsymbol{c} \cos \frac{1}{a}},
$$

daraus

$$
\operatorname{tg} B L,=\frac{l \boldsymbol{c} \sin \alpha}{k \boldsymbol{b}+l \boldsymbol{c} \cos \alpha}
$$

und

$$
\frac{\sin B L,}{\sin \left(\alpha-B L_{,}\right)}=\frac{\sin B L,}{\sin C L_{,}}=\frac{l \boldsymbol{c}}{k \boldsymbol{b}}
$$

Dividirt man (21) durch (17), so erhält man das anharmonische Verhältniss der vier Pule $\{B L, L C\}$, welches ist:

$$
\sin B L, \div \frac{\sin B L}{\sin C L,}=\frac{l}{\sin C L}=\frac{l}{k} .
$$

Die Ableitung specieller Formeln für die Systeme mit grösserer symmetrie ist leicht. Wir wollen uns hier auf die Belrachlung des rhomboëdrischen Systems beschränken.

\section{Rhomboëdrisches System.}

In diesem Systeme werden die Normalen zu den Ebenen der primïren trigonalen Pyramide oder des Rhomboëders zu $\Lambda$ xenstrahılen gewähll und die dreizühlige $\Lambda$ xe als Parameterstralıl (111).

Daher $\alpha=\beta=\gamma ; a=b=e$ werde glcich 1 gesetzt.

1)ie Gleichungen ( $(\xi)$ werden:

$$
\left.\begin{array}{ll}
O P \cos \lambda=h+(k+l) \cos \alpha & =h(1-\cos \alpha)+\theta \cos \alpha \\
O P \cos \mu & =h(1-\cos \alpha)+\theta \cos \alpha \\
O P \cos \nu & =l(1-\cos \alpha)+\theta \cos \alpha
\end{array}\right\},
$$


wo

$$
\theta=h+k+l \text {. }
$$

Ferner $O P^{2}=\Sigma h^{2}+2 \cos \alpha \Sigma h l=\theta^{2} \cos \alpha+(1-\cos \alpha) \Sigma h^{2}$.

lund

$\triangle 1$ wird:

$$
\left.\begin{array}{l}
\cos \lambda=\frac{h(1-\cos \alpha)+\theta \cos \alpha}{\sqrt{\Sigma} h^{2}+2 \cos a \Sigma l} \\
\cos \mu=\frac{k(1-\cos \alpha)+\theta \cos \alpha}{\sqrt{\Sigma h^{2}+2 \cos \alpha \Sigma l l}} \\
\cos \nu=\frac{l(1-\cos \alpha)+\theta \cos \alpha}{\sqrt{\Sigma} h^{2}+2 \cos \alpha \Sigma l}
\end{array}\right\},
$$

$$
\begin{gathered}
(1-\cos \alpha)^{2}(1+2 \cos \alpha)-\left(1-\cos ^{2} \alpha\right) \Sigma \cos ^{2} \lambda \\
+2 \cos \alpha(1-\cos \alpha) \leq \cos \lambda \cos \mu
\end{gathered}
$$

1)a dies gleich Null ist, erhalten wir als Relation zwischen den Richtungscosinus:

$(1+\cos \alpha) \Sigma \cos ^{2} \lambda-2 \cos \alpha \Sigma \cos \lambda \cos \mu=(1-\cos \alpha)(1+2 \cos \alpha) \cdot(26)$

Wenn der Strahl mit der dreizühligen $\Lambda$ xe coïndicirt, so wird $\lambda=\|$ $=y=D=111: 100$, dann reducirt sich Gleichung (26) auf:

Daher:

$$
\begin{aligned}
3(1-\cos a) \cos ^{2} D & =(1-\cos \alpha)(1+2 \cos \alpha) ; \\
\therefore 3 \cos ^{2} D & =1+2 \cos \alpha .
\end{aligned}
$$

$$
2 \cos \alpha=\frac{2-\operatorname{tg}^{2} D}{i+\operatorname{tg}^{2} D} \text {. }
$$

Ferner:

$$
3 \sin ^{2} D=2(1-\cos \alpha)=4 \sin ^{2} \frac{\alpha}{2} .
$$

)iese letzle Gleichung ist ïquivalent der wohlbckannten Formel:

$$
\sin \frac{r^{\prime} r}{2}=\sin 60^{\circ} \sin D
$$

wo $r$ und $r^{\prime}$ zwei Pole des primüren Rhomboëders sind.

Der Ausdruck (14) für den Cosinus des Winkels zwischen den Strablen $(h k l)$ und $\left(h_{1} k_{1} l_{1}\right)$ wird:

$$
\cos q=\frac{\Sigma h h_{1}+\cos \alpha \Sigma\left(k l_{1}+k_{1} l\right)}{\sqrt{\bar{\Sigma} h^{2}}+2 \cos \alpha \Sigma \overline{k l} \sqrt{\bar{\Sigma} h_{1}^{2}+2 \cos \alpha \Sigma k_{1} l_{1}}} .
$$

Wenn $\cos \alpha$ durch sein in (28) gegebenes Aequivalent crselzt wird, so erhalten wir die folgenden Ausdrücke für den Cosinus:

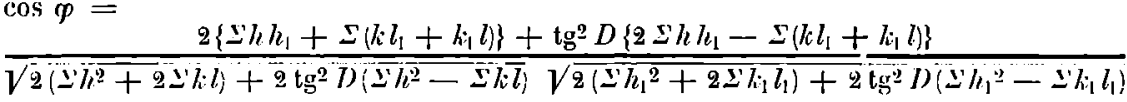

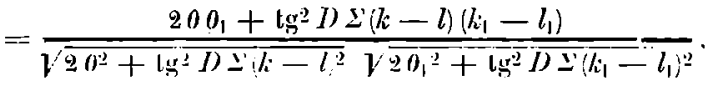


wo

$$
\theta_{1}=h_{1}+k_{1}+l_{1} .
$$

Mittelst der oben gegebenen Gleichungen und der Relationen der sphïrischen Dreiecke, welche $P$ mit den $\Lambda$ xenpolen und mit den in Fig. 2 bezeichneten Polen $C, N, m, m^{\prime}, m^{\prime \prime}$, $a^{\prime}$ u. s. w. bildet, künnen wir leicht die allgemein für die Auflüsung der auf rhomboëdrische Krystalle bezüglichen Probleme gebräuchlichen Relationen aufstellen.

Oder, wenn man die allgemeinen Gleichungen (30) und (31) anwendet, und wenn $C$ bedeutet (111), so erhält

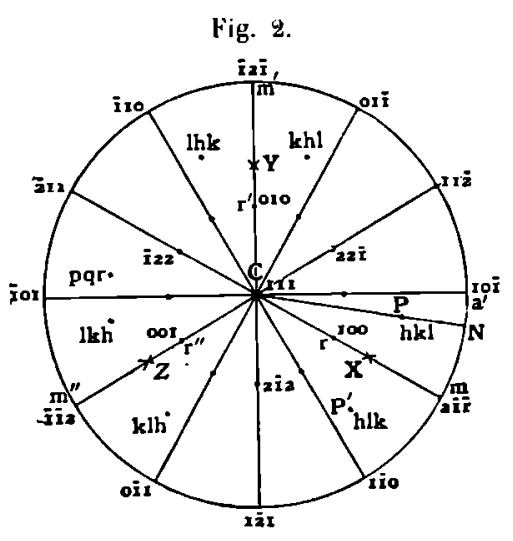
man:

$$
\begin{aligned}
\cos C \boldsymbol{P} & =\frac{\theta \sqrt{ }(1+2 \cos \alpha)}{\sqrt{3\left\{\Sigma l^{2}+2 \cos \alpha \Sigma k l\right\}}} \\
& =\frac{\theta \sqrt{2}}{\sqrt{2 \theta^{2}+\operatorname{tg}^{2} D \Sigma\left(k-l^{2}\right.}} .
\end{aligned}
$$

Trạsformirt man die erste Gleichung, so erhält man:

$$
\operatorname{tg} C P=\frac{v \overline{(1-\cos \alpha) \Sigma(k-l)^{2}}}{\theta \sqrt{(1+2 \cos \alpha)}} \text {. }
$$

Durch Transformation der zweiten Gleichung dagegen ergiebt sich:

$$
\operatorname{tg} C P=\frac{\sqrt{\Sigma(k-l)^{2}}}{\theta \sqrt{2}} \operatorname{tg} D
$$

und von dicser Formel kann bewiesen werden, dass sie sich ergiebt, wenn man $\cos \alpha$ in (33) ersetzt durch sein Aequivalent in Gliedern von $\operatorname{tg} D$.

Es seien ferner $2 \xi, 2 \eta$ die Winkel der stumpfen, respective der scharfen Polkante eines Skalenoëders $\{h l c l\}$ und $2 \zeta$ derjenige seiner Miltelkanten, so haben wir:

$$
\begin{gathered}
\cos 2 \xi=\cos (h k l: h l l)=\frac{h 2+2 k l+\cos \alpha\left\{\left(\theta^{2}-\left(h^{2}+2 k l\right)\right\}\right.}{\Sigma^{2} h^{2}+2 \cos \alpha \Sigma l^{\prime} l} \\
=\frac{\left.2 \theta^{2}+\operatorname{tg}^{2} D\right)\left\{3\left(h^{2}+2 k l\right)-\theta^{2}\right\}}{2 \theta^{2}+\operatorname{tg}^{2} D \Sigma(k-l)^{2}} . \\
\text { Daher } 2 \sin ^{2} \xi=\frac{2(k-l)^{2} \sin ^{2} \frac{\alpha}{2}}{\Sigma h^{2}+2 \cos \alpha \Sigma k l}=\frac{3(k-l)^{2} \lg ^{2} D}{2 \theta^{2}+\frac{D}{\operatorname{tg}^{2} D \Sigma(h-l)^{2}}} .
\end{gathered}
$$


Ebenso

$$
\begin{aligned}
& \cos 2 \eta=\cos (h k l: k h l)=\frac{l^{2}+2 h k+\cos \left\{\theta^{2}-\left(l^{2}-2 h h\right)\right\}}{\Sigma h^{2}+2 \cos \alpha} \\
& =\frac{2 \theta^{2}+\operatorname{tg}^{2} D\left\{3\left(l^{2}+h k\right)-\theta^{2}\right\}}{2 \theta^{2}+\operatorname{tg}^{2} D \Sigma(k-l)^{2}} \text {. } \\
& \therefore 2 \sin ^{2} \eta=\frac{2(h-k)^{2} \sin ^{2} \frac{\alpha}{2}}{\Sigma h^{2}+2 \cos \alpha \Sigma h l}=\frac{3(h-k)^{2} \operatorname{tg}^{2} D}{2 \theta^{2}+\operatorname{tg}^{2} D \Sigma(k-l)^{2}} .
\end{aligned}
$$

Und

$$
\begin{aligned}
& \cos 2 \zeta=\cos (h k l: \bar{l} \bar{l} \bar{h})=\text { 二- } \frac{\left(k^{2}+2 h l\right)-\cos \alpha\left\{\theta^{2}-\left(k^{2}+2 h l\right)\right\}}{\Sigma h^{2}+2 \cos \alpha \Sigma l} \\
& \begin{array}{c}
=2 \theta^{2}-\operatorname{tg}^{2} D\left\{3\left(k^{2}+2 h l\right)-\theta^{2}\right\} \\
2 \theta^{2}+\operatorname{tg}^{2} D \mathrm{~s}(k-l)^{2}
\end{array} .
\end{aligned}
$$

Daher

$$
2 \cos ^{2} \zeta=\frac{2(h-l)^{2} \sin ^{2} \frac{\alpha}{2}}{\Sigma h^{2}+2 \cos \alpha \Sigma \overline{k l}}=\frac{3(h-l)^{2} \operatorname{tg}^{2} D}{2 \theta^{2}+\operatorname{tg}^{2} D \Sigma(k-l)} \text {. }
$$

Nun ist jeder der Winkel $\xi$, $\eta$ und $\zeta$ kleiner als $90^{\circ}$, da jeder der Winkel an den Skalenoëderkanten kleiner als $180^{\circ}$ ist. Nehmen wir daher, was wir $\mathrm{zu}$ thun berechtigt sind, $h, k$ und $l$ in der Reihenfolge mit abnehmender Grösse und ihre Summe grösser als Eins an, so müssen die positiven Werthe genommen werden, wenn die Quadratwurzeln der Gleichungen (36), (38) und (40) ausgezogen werden. Wir erhalten daher:

$$
\begin{aligned}
& \sin \xi=\frac{(k-l) \sin \frac{\alpha}{2}}{\sqrt{\Sigma} \frac{1}{h^{2}+2} \frac{(k-l) \sqrt{3} \operatorname{tg} D}{\cos \alpha \Sigma} k l}=\frac{\left(\operatorname{tg}^{2} D \Sigma(k-l)^{2}\right.}{\sqrt{4 \theta^{2}+2}} ; \\
& \sin \eta=-\frac{(h-k) \sin \frac{\alpha}{2}}{\sqrt{\Sigma} h^{2}+2 \cos \alpha \dot{\Sigma} \overline{k l}}=\frac{(h-k) \sqrt{3} \operatorname{tg} D}{\sqrt{4} \theta^{2}+2 \operatorname{tg}^{2} D \Sigma(k-l)^{2}} ;
\end{aligned}
$$

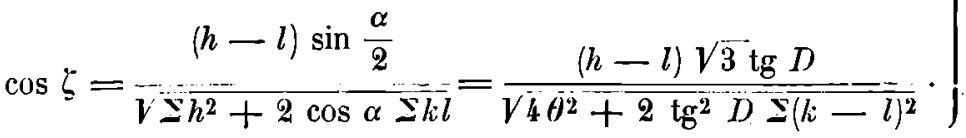

$$
\begin{aligned}
& \frac{\sin \xi}{k-l}=\frac{\sin \eta}{h-k}=\frac{\cos \zeta}{h-l}=\frac{\sin \frac{\alpha}{2}}{\sqrt{\Sigma} h^{2}+2 \cos \alpha \Sigma \overline{k l}}
\end{aligned}
$$

$=[$ durch T'ransformation aus (32)]:

$$
\begin{gathered}
\frac{\sqrt{3} \sin C P}{\sqrt{2 \Sigma}(k-l)^{2}}=\frac{\sqrt{3} \operatorname{tg} D}{\sqrt{4 \theta^{2}+2 \operatorname{tg}^{2} D \Sigma(h-l)^{2}}} \\
=[\operatorname{aus}(32)] \frac{\sqrt{3} \operatorname{tg} D \cos C P}{2 \theta}
\end{gathered}
$$

und

$$
\sin \xi+\sin \eta=\cos \xi \text {. }
$$

